\title{
Fuzzy-PID control algorithm of a loop reactor for microbial corrosion testing
}

\author{
D. Rangel-Miranda ${ }^{1}$, D. Alaniz-Lumbreras ${ }^{2}$, V.M. Castaño ${ }^{1 *}$ \\ ${ }^{1}$ Centro de Física Aplicada y Tecnología Avanzada, Universidad Nacional Autónoma de México, Boulevard Juriquilla 3001, \\ Querétaro 76230, México \\ ${ }^{2}$ Unidad Académica de Ingeniería Eléctrica, Universidad Autónoma de Zacatecas, Zacatecas, Zacatecas 98000, México \\ *Corresponding authorE-mail: meneses@unam.mx
}

Copyright $\odot 2015$ V.M. Castaño et al. This is an open access article distributed under the Creative Commons Attribution License, which permits unrestricted use, distribution, and reproduction in any medium, provided the original work is properly cited.

\begin{abstract}
The thermal control of loop reactor utilized to run hydrodynamic tests of microbical corrosion, where full control of the temperature is crucial, is presented. Since the accuracy of the temperature is critical along the pipe trajectory for the microbial culture, it must be controlled with an accuracy of $\pm 0.5^{\circ} \mathrm{C}$, which is achieved by an implemented fuzzy-PID (Proportional Integral and Derivative) control algorithm, capable to provide the accuracy at the temperature range required. The system counts with an especially-designed software to program the desired temperature. Several tests were carried out at different temperatures and water volumes to characterize the rising time and thermal inertia presented by the system. As a result, the performance and power consumption were notability improved.
\end{abstract}

Keywords: UZZY-PID Algorithm; Intelligent Thermal Control; Loop Reactor Process; Microbial Corrosion Testing.

\section{Introduction}

It is well known that a fluid circulating in a loop reactor for corrosion testing is one of the most difficult systems in order to model and analyze in terms of its dynamic thermal behavior. In general, for compressible fluids, it is essential take into account spatial and temporal variation of the physical magnitudes [1]. These are the so-called systems with distributed parameters, and their analysis by dynamic models is based on complex partial differential equations [2]. The fundamental relationships that govern these phenomena are summarized in the Navier-Stokes equations. On the other hand, the changes in the descriptive variables of the fluids originate changes in their temperature [3]. However, there is a very important subgroup of fluid-dynamics systems that can be described by using models of concentrated parameters [4]. This means that, in certain regions, the physical magnitudes can be considered no only uniforms but also having temporal variation [5]. These characteristics allow its dynamic description by ordinary differential equations and such subset of systems is usually designated as Hydraulic Systems. In these cases, the fluid is in general either water or oil with low compressibility, higher work pressures and low speeds, even though for important volumes in the flow, a series of simplified hypotheses that allow them to be treated as hydraulic circuits, can be assumed. On the order hand, many intelligent systems have been applied to industrial distributions of fluids such as oil, gas or water either for plant monitoring [6] or faults in critical parameters, as the lost circulation problem while drilling [7].

Accordingly, this work proposes to apply a new intelligent algorithm, by modifying previous models [8] and composed by both a classic control type PID and a fuzzy controller [9], as a novel way to maintain in optimal conditions of operation and stability [10] the flow and temperature in an experimental loop reactor for microbial corrosion testing [11]. 


\section{Materials and methods}

\subsection{Experimental setup reactor system}

Fig. 1a shows a schematic diagram of the reactor, fabricated from a PVC pipe of $0.0508 \mathrm{~m}$ inner diameter, $0.005 \mathrm{~m}$ thickness wall and $7.0 \mathrm{~m}$ total length. The loop reactor consists of two cylindrical tanks made of polypropylene and 100 liters of capacity which storage a given volume of water.

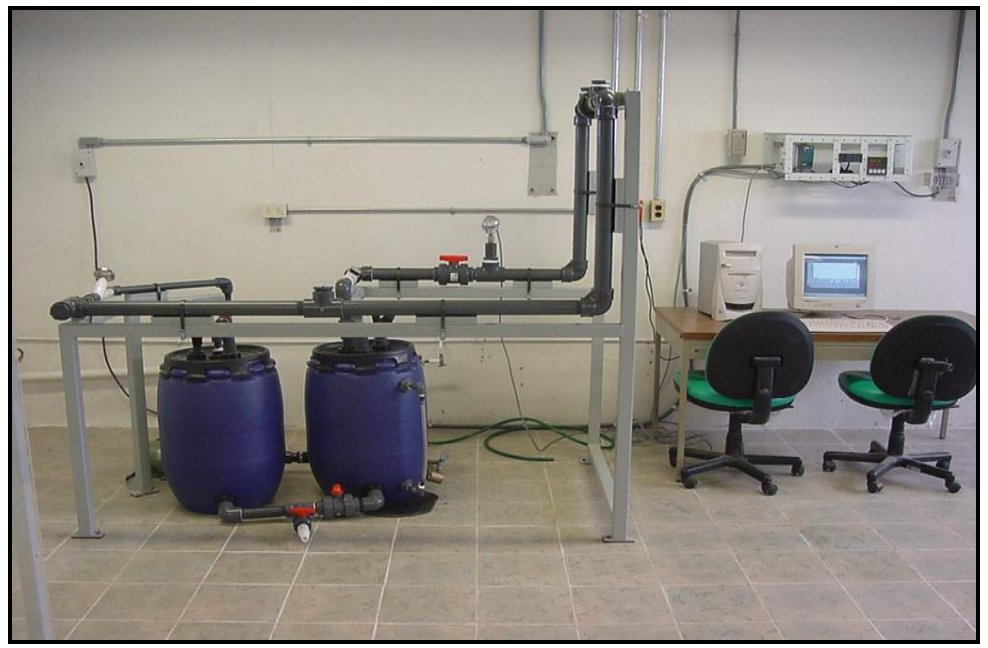

Fig. 1: A) Loop Reactor System Designed and Built for Corrosion Testing.

The microbial culture (of the bacillus stearothermophilus type) and normalized metal samples are introduced into the containers at desired temperature, expecting that the microbial culture attacks the metal samples [12]. Also, the tube has some probe insertion ports in order to set strategically the samples. Temperatures in loop reactor were measured by a RTD platinum sensor (PT100) protected with stainless steel. The temperature sensor is placed in a probe insertion port as can be observed in the Fig. 1b. The aim of this system is to control the behavior temperature of the fluid along the tubular zone. The corrosion temperature for the several microbial cultures is ranged from $60{ }^{\circ} \mathrm{C}$ to $80^{\circ} \mathrm{C} \pm 0.5{ }^{\circ} \mathrm{C}$ [13]. The closed-loop control strategy contains the fuzzy logic PID algorithm based on a classical control scheme and fuzzy logic control.

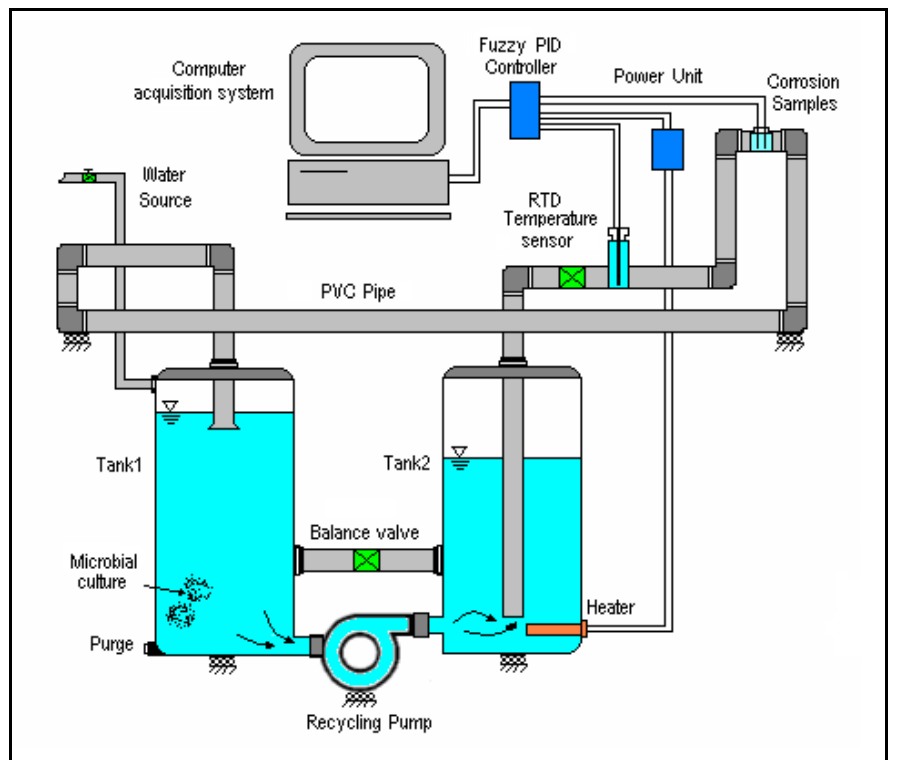

Fig. 1: B) Schematic Diagram of the Loop Reactor by Using a Fuzzy-PID Control Algorithm.

The heater device is a $16.37 \mathrm{~W}$ resistance driven by a power electronic interface. At each running, the heater increases the temperature directly from room conditions to the set-point. The system was constructed according to an engineering approach [14] where is usual to analyze the system by mass balance equations, using the volumetric flow instead of mass flow. Also, static friction and secondary thermal effects can be neglected. It is important to clarify that this point 
of view will be validated considering short variations around their static operation points as was proposed by Trilleros [15] where the variables in this hydraulic system represent volume, pressure and flow.

\subsection{Theoretical background}

The first considered phenomenon is the hydraulic resistance. This phenomenon produces a fall in the pressure pipe due the loss of energy (internal friction in the fluid and from the fluid to the walls of the pipe interacting with it). In general, the pressure in the fall is related to the caudal just as:

$f(\Delta P, Q)=0$

In this case, the linear relation is:

$\Delta P-Q R=0$

Where:

$\Delta P=$ differential pressure between two po ints

$Q=$ flow

$R=$ resis $\tan$ ce is opposed by the conduit

Other essential phenomenon that appears in the hydraulic systems is the storage of potential energy in a fluid. This energy can be the potential energy of the gravitational field, as it has been described [16]. Since we work with volumetric flow, we assume the fluid as incompressible and the elasticity should be attributed to the walls. Both cases of storage potential energy (gravitational or elastic) represent the so-called hydraulic capacitor which basically links the pressure and volume of fluid by mean a constitutive relation, given as

$f(P, V)=0$

For the linear case:

$V-C P=0$

Where $C$ is the so-called capacity. In both linear and nonlinear cases, we must add another fundamental constitutive relation that dynamically links the caudal with the accumulated volume as:

$$
\frac{d V}{d t}=Q
$$

In a tank of constant area $A$ we know that the pressure in the bottom tank due to water column is given by

$$
P=\rho g h=\frac{\rho g}{A} V
$$

As a result:

$$
C=\frac{A}{\rho g}
$$

If the cross section of a tank is not constant, the function that relates the pressure and the volume is nonlinear, because the relation between $V$ and $P$ is nonlinear too.

Also, those constitutive relations link both the presence of dissipation phenomenon and energy storage in the fluids. In that sense, the hydraulic circuits have found structural relations that are completely analog to Kirchhoff's laws. In this case, much of the thermodynamic behaviour involves processes related to the heat concept and energy conservation.

Based on a classical approach, without considering irreversible process, we distinguish three types of elemental phenomena: generation, storage and heat transfer. The main variables are temperature, heat amount and flow of heat transfer. In general, the temperature is spatially distributed and the models of thermal systems habitually result in distributed parameters systems and are presented by partial differential equations. 
A similar reduced distributed parameters model of a loop reactor has been developed [17] by making some reasonable assumptions. The model consists of six partial differential equations and the corresponding boundary condition. For the $i$ th section of the circulation loop reactor, the model equation can be represented as:

$$
\begin{aligned}
& \frac{\partial T_{i}}{\partial t}=f i\left(T_{i} \frac{\partial T_{i}}{\partial z}, \frac{\partial^{2} T_{i}}{\partial z^{2}}, T a, x_{i}\right) \\
& 0=g_{i}\left(x_{i}, \frac{\partial x_{i}}{\partial z}, \frac{\partial^{2} T_{i}}{\partial z^{2}}, T_{i}\right)
\end{aligned}
$$

\section{Where}

$i=I, L, J$ the subscripts $I, L$ and $J$ denote the inner tube of the exchanger, the reactor loop and the wall of the heat exchanger respectively; $f$ and $g$ are nonlinear functions; $T, x, z$ and $t$ denote temperature, concentration, axial coordinate and time, respectively. Ta = room temperature .

Extensive dynamic simulations show that the model can to describe the steady state and the dynamic behavior of the loop reactor. We have made simplifications that are customary in engineering problems in order to eliminate the space as an absolute variable [18]. The simplifications consist on dividing the space in several regions and to consider that each one has a uniform temperature.

By considering exclusively energy storage, it results in a heat balance of a loop reactor during a thermodynamic process. In this way, we have always a fundamental structural relation as follows:

$$
Q_{\text {net }}-Q_{\text {generated }}-Q_{\text {received }}+Q_{\text {delivered }}=0
$$

Besides the constitutive relations associated to the transport phenomena and heat storage, we can find the structural relations of the system. We have mentioned one that is an expression of heat balance that defines the quantity of storage heat. In general, we work with its analog version, expressed as flow balance:

$$
\varphi_{\text {net }}-\varphi_{\text {generated }}-\varphi_{\text {flow -in }}+\varphi_{\text {deliver }}+\varphi_{\text {flow -out }}=0
$$

Fig. 2 shows a simplified low-order model that includes these parameters.

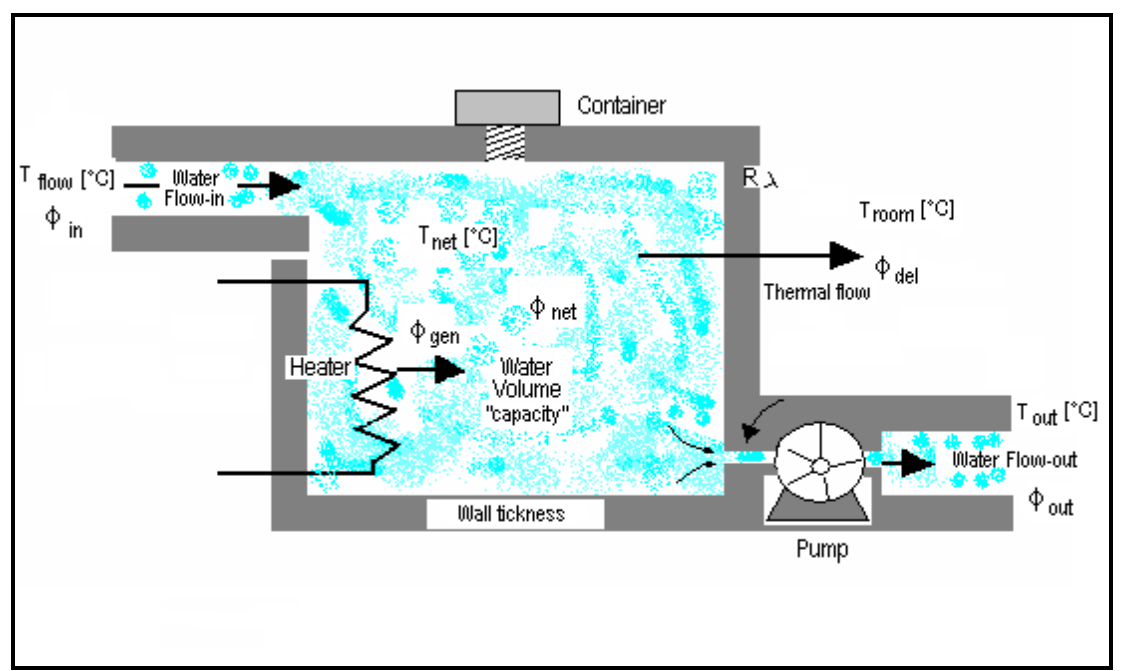

Fig. 2: Simplified Concentrated Parameters Low-Order Model for the Loop Reactor.

So, we can write the following structural relation

$$
\varphi_{\text {net }}-\varphi_{\text {in }}-\varphi_{\text {gen }}+\varphi_{\text {del }}+\varphi_{\text {out }}=0
$$

Which is included in the corresponding constitutive relations, related to the mass transport phenomena, the conduction of heat to the environment and the heat generation through the heater resistor. 


\subsection{Fuzzy-PID control algorithm}

Fuzzy-PID control can be considered as a dynamical expert system where expert knowledge about the system is encoded in a Fuzzy Rule-Base (FRB), which works in order to optimize the response of a classical PID control algorithm. The associated Fuzzy Inference Engine (FIE) is the tool for performing the fuzzy inferences [19]. Information from the system leads to fuzzy-decision-making via the firing of rules in the FRB through the application of fuzzy set-theoretical calculations. The decisions of the fuzzy expert systems are converted to control actions by mean the temperature actuator. The design flexibility involves a large number of choices for the various models of the fuzzy logic and related set-theoretical operators, inference schemes, and defuzzification maps. The general combinatorial complexity of all these fuzzy substructures precludes the possibility of the existence of only a single, or a standardized fuzzy controller format (such as it is available in linear PID controllers). The particular choice of these design parameters, based on heuristics, is clearly stated below.

In general, we design a fuzzy inference system based on the past known behavior of a target system. The fuzzy system is then expected to be able to reproduce the behavior of the target system. We consider how to construct a fuzzy inference system for a specific application by using modified triangular membership [20]. The standard method for constructing a fuzzy inference system [21] is a process called fuzzy modeling, it is as follows:

The rule structure of a fuzzy inference system makes it easy to incorporate human expertise about the target system directly into the modeling process. Namely, fuzzy modeling takes the advantage of "domain knowledge" that might not be easily or directly employed in other modeling approaches. When the input-output data of the target system is available, conventional system identification techniques can be used for fuzzy modeling. In other words, the use of numerical data also plays an important role in fuzzy modeling, just as in other mathematical modeling methods.

Conceptually, fuzzy modeling can be pursued in two stages [22], which are not totally disjoint to each other. The first stage is the identification of the surface structure, which include the following tasks:

1) Select relevant input and output variables.

2) Choose a specific type of inference system.

3) Determine the number of linguistic terms associated to each input and output variables.

4) Design a collection of fuzzy if-then rules.

To accomplish the preceding tasks, we rely on our own knowledge such as common sense, simple physical laws and so on of the target system, information provided by human experts who are familiar to the target system, or simply by trial and error.

After the first stage of fuzzy modeling, we obtain a rule that can more or less describe the behavior of the target system by means of linguistic terms. The meaning of these linguistic terms is determined in the second stage: the identification of deep structure, which determines the Membership Functions (MF) of each linguistic term, the identification of deep structure includes the following tasks:

1) Choose an appropriate family of parameterized MFs.

2) Interview human experts familiar with the target systems or experimental trials to determine the parameters of the MFs used in the rule base.

3) Refine the parameters of the MFs using regression and optimization techniques.

Tasks 1 and 2 assume the availability of human experts, whereas task 3 assumes the availability of a desired inputoutput data set.

\subsection{A typical structure of takagi-sugeno (TS) fuzzy controllers}

The typical Takagi-Sugeno (T-S) controllers [23] have two inputs and one output. The input variables are error and rate change of error of the system output with respect to output setpoint. They are designated as follows:

$e(n T)=S P(n T)-y(n T)$
$r(n T)=e(n T)-e(n T-T)$

Where $n$ is a positive integer, $T$ sampling time and $S P(n T)$ setpoint signal of the system output. We denote $e(n T), r(n T)$ and $y(n T)$ as error, rate of error and system output, respectively. The two input variables are fuzzified by the same input fuzzy sets. The fuzzy controller employed in this work has the following form of T-S fuzzy rules:

if $e(n T)$ is $E i$ AND $r(n T)$ is $R j$ THEN $\Delta u(n T)=a_{i, j} e(n T)+b_{i, j} r(n T)$

Where $\Delta u(n T)$ is the contribution of this rule to the change of the fuzzy controller output and $a_{i, j}$ and $b_{i, j}$ are design parameters [24]. 


\subsection{Fuzzy logic for fine tuning PID control}

This novel technique allows adjusting the quickness, slowness and overshooting over the thermal response in a circulated water system [25]. The set-point in the heating process can be achieved in less time than the classical PID control. Also, slowness and overshoot can be modified from the thermal response at first stage of the process control by the PID self-tuning. This action is very difficult because of the existence of distributed parameters such as heat dissipation along the tube in the loop reactor. So, should be used a manual tuning when the process does not allows using the self-tuning mode by adjusting the PID via approximation as follows:

To introduce the set point that requires the process. Fix $\mathrm{Bp}=10 \%$ with $\mathrm{t}=0$ and $\mathrm{td}=0$. Observe the process performance. If the thermal response oscillates, to increase $b P$ until stabilization.

If the thermal response in the loop reactor is slow, to decrease $b P$, then to observe the behavior.

Since the system is working only in a proportional mode, it will produce an offset error. When the oscillation is minimum by increasing $b P$ in one or two points, it determines the integral action $t i$.

To select a value of $t i$ big enough for getting a behavior almost perfect, it takes around 30 minutes. After that, decrease slowly $t i 5$ in $5 \mathrm{~min}$, stops and for $1 \mathrm{~min}$ stops and check the offset error that has been corrected, then generate disturbances in the process by changing the set-point and the adjustment will be corrected if the oscillation damping goes from 4 to 1 .

To put the time $t d$ in range $1 / 5$ to $1 / 10$ of the adjusted time in $t i$.

In Table 1, the manual tune was implemented by adjusting the PID parameters as follows:

Table 1: PID Parameters in Manual-Tuning Mode.

\begin{tabular}{ll}
\hline PID Parameters & Value \\
\hline Proportional Band & $8 \%$ \\
Integral time & $2.8[\mathrm{~min}]$ \\
Derivate Time & $28[\mathrm{sec}]$ \\
Cycle Time & $60[\mathrm{sec}]$ \\
\hline
\end{tabular}

The digital control algorithm does the control of the system by the implementation of classical PID control and optimization of the fuzzy rules-based together [26]. The optimal performance of the system was obtained by increasing the response time while decreasing the overshoot in thermal behavior. The linguistic rules, fuzzification and defuzzification process are summarized in the Table 2.

Table 2: Sumary of Linguistic Rules and the Fuzzy Control Actions.

\begin{tabular}{lll}
\hline Fast response & Slow response & Overshoot \\
\hline Nothing & Nothing & Nothing \\
Some & Some & Some \\
Some More & Some More & Some More \\
Much More & Much More & Much More \\
Much Much More & Much Much More & Much Much More \\
\hline
\end{tabular}

\section{Results}

The fluid in the loop reactor was drinkable water and the microbial culture was introduced there. We ran several industrial tests in the loop reactor using a water flow rate of approximately $1381 / \mathrm{min}$ to determine the dynamical thermal behavior by a fuzzy-PID control algorithm [27]. First, were made experimental trials at $60^{\circ} \mathrm{C}$ and different volumes of water $(60,70$ and 80 liters). Fig. 3 shows the rising time obtained by the optimized fuzzy-PID control algorithm at $60^{\circ} \mathrm{C}$ as a function of time. For the trials carried out using 60, 70 and 80 liters of water is observed that the programmed temperature reaches the set point from 150 to 240 minutes. 


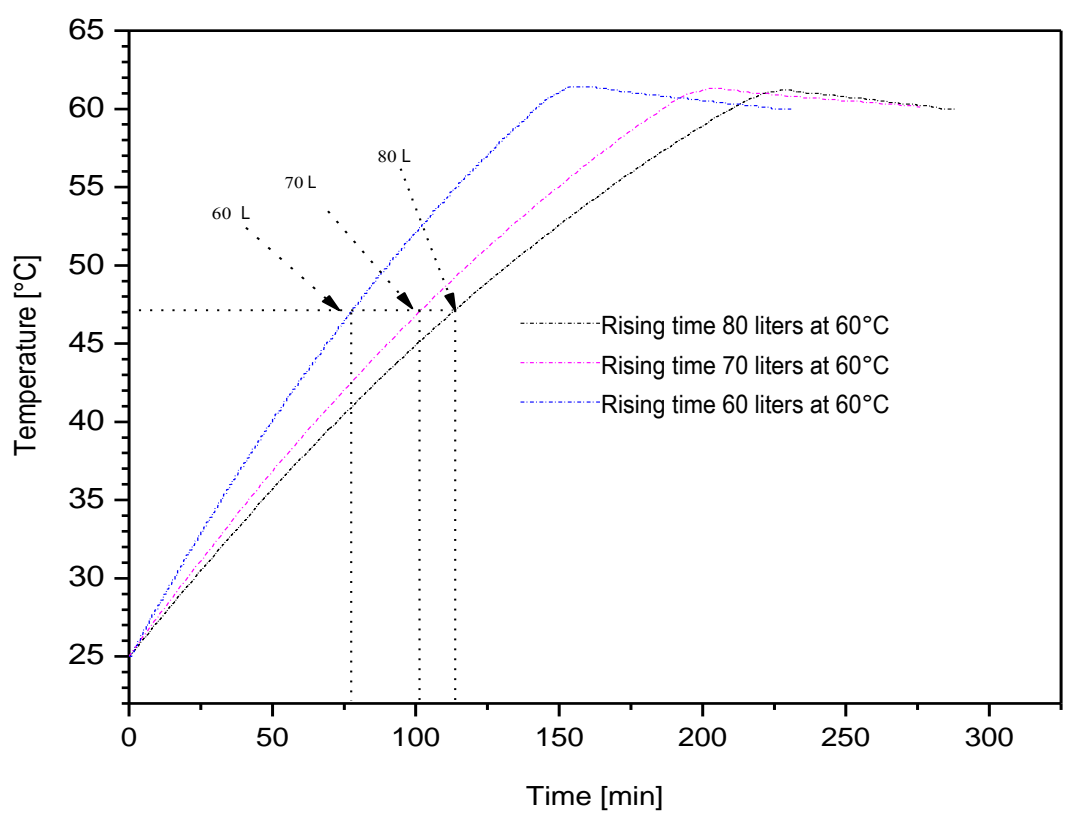

Fig. 3: The Rising Time by Using a Fuzzy-PID Control Algorithm at $60^{\circ} \mathrm{C}$ with 60,70 and 80 Liters Water Volumes.

In this figure we observe that each temperature should be a linear response of a temperature ramp programmed algorithm. According to the quantity of fluid into the reactor, the temperature is raised linearly. However, we can consider that the response is nonlinear, since the system arrived smoothly to the programmed temperature. When we applied exclusively classical PID control, there existed an overshoot of $2.5^{\circ} \mathrm{C}$ and the response was slow. The overshoot affects the conditions of the thermal behavior and can damage the microbial culture. When the fuzzy PID control is applied, we reduce significantly the overshoot $1.3^{\circ} \mathrm{C}$, almost $50 \%$ by considering this system as a low-order system. So that we can say that the setting times at the steady state of temperature were 230, 275 and 300 minutes at 60,70 and 80 liters, respectively. The accuracy temperature in steady state was $60 \pm 0.2^{\circ} \mathrm{C}$.

In the second test were utilized similar volumes $(60,70$ and $80 \mathrm{l})$ at a water temperature of $70^{\circ} \mathrm{C}$. The responses are similar but, in general, increasing their nonlinearity. Fig. 4 shows the rising time at $70^{\circ} \mathrm{C}$.

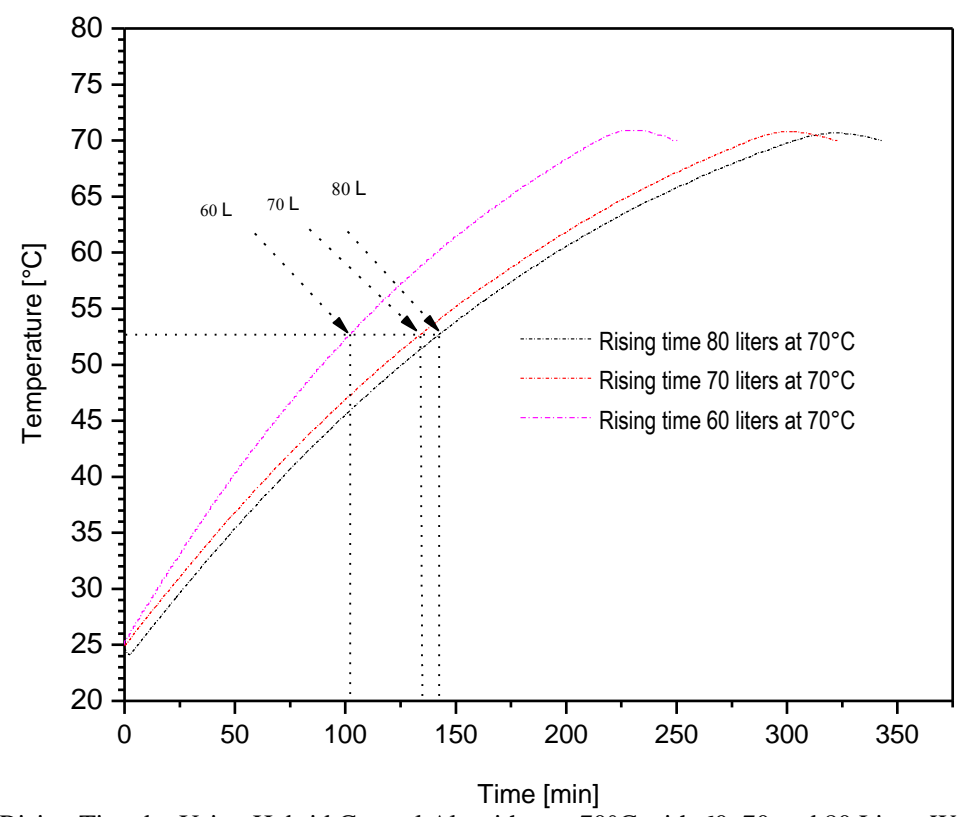

Fig. 4: The Rising Time by Using Hybrid Control Algorithm at $70^{\circ} \mathrm{C}$ with 60,70 and 80 Liters Water Volumes.

A logarithmic fitting function is shown. It is important to point that the responses are nonlinear too.

The transitory response is similar in three cases, but each one has been different overshoot. We assumed the loop reactor system as a low-order thermal system in steady state. The overshoot is only $0.9^{\circ} \mathrm{C}$ in every case. Additionally, we optimized the responses by applying the based-rule fuzzy PID control algorithm, which decreased significantly the rising time and overshoot. The steady state temperature was $70 \pm 0.3^{\circ} \mathrm{C}$.

Fig. 5 shows the thermal behavior responses at $80^{\circ} \mathrm{C}$. In the third test, we utilized similar volumes water and microbial culture. The rising time of the reactor system is increased when the hydraulic capacitance is bigger than once. 


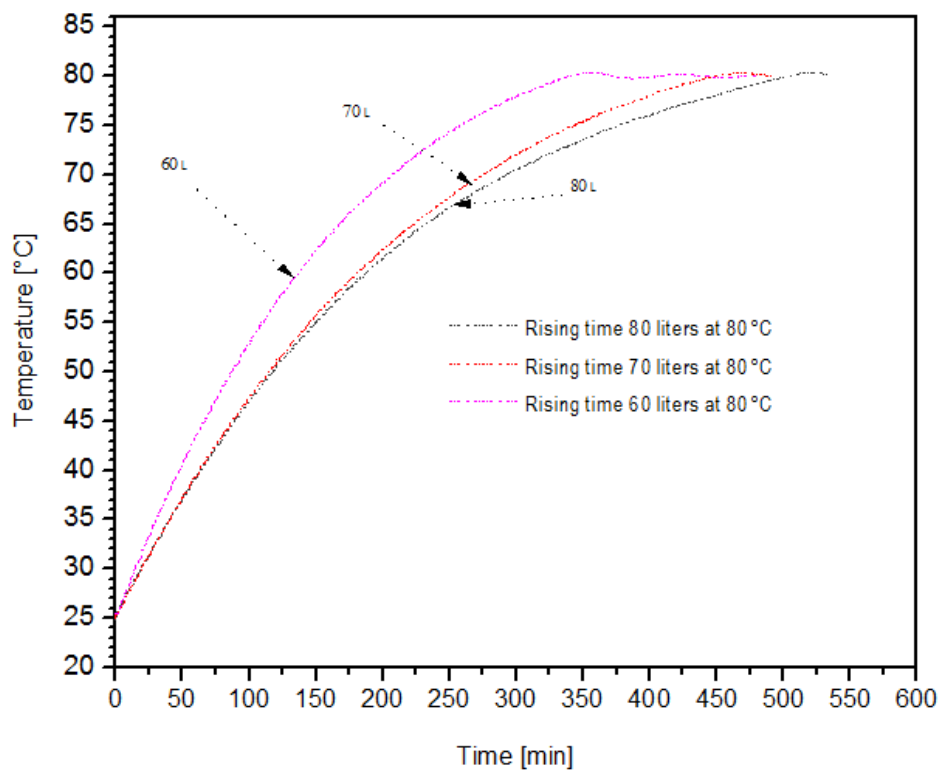

Fig. 5: The Rising Time by Using Hybrid Control Algorithm at $80^{\circ} \mathrm{C}$ Over 60,70 aAnd 80 Liters Water Volumes.

The thermal inertia should indicate that the behavior system is like a double series-parallel first-order electrical system, since an intrinsic hydraulic capacitance and a thermal conductive resistance (between the heater and the water together) forms an analog equivalent $R C$ electric circuit and the pipe wall with environment. When the temperature and water volumes are higher than in other cases, the time-constant $[\tau]$ is increased nonlinearity, so that the values for the volumes 60, 70 and 80 liters were 132, 177 and 182 minutes, respectively. The overshoot is $0.4^{\circ} \mathrm{C}$ and the rising times are modified by the implementation of the rule-based fuzzy control algorithm. The accuracy in the steady-state temperature was possible $80 \pm 0.4^{\circ} \mathrm{C}$ because of the use of a fuzzy logic control in order to optimize the thermal behavior of a loop reactor by a single classical PID control.

The numerical fit, for intrinsic thermal inertia of the loop reactor is:

$T(t)=T_{0}+\left(1-e^{-\frac{t}{\tau}}\right) \Delta T$

Which corresponds to measured responses in thermal intrinsic inertia (without temperature control) shown in the Fig. 6.

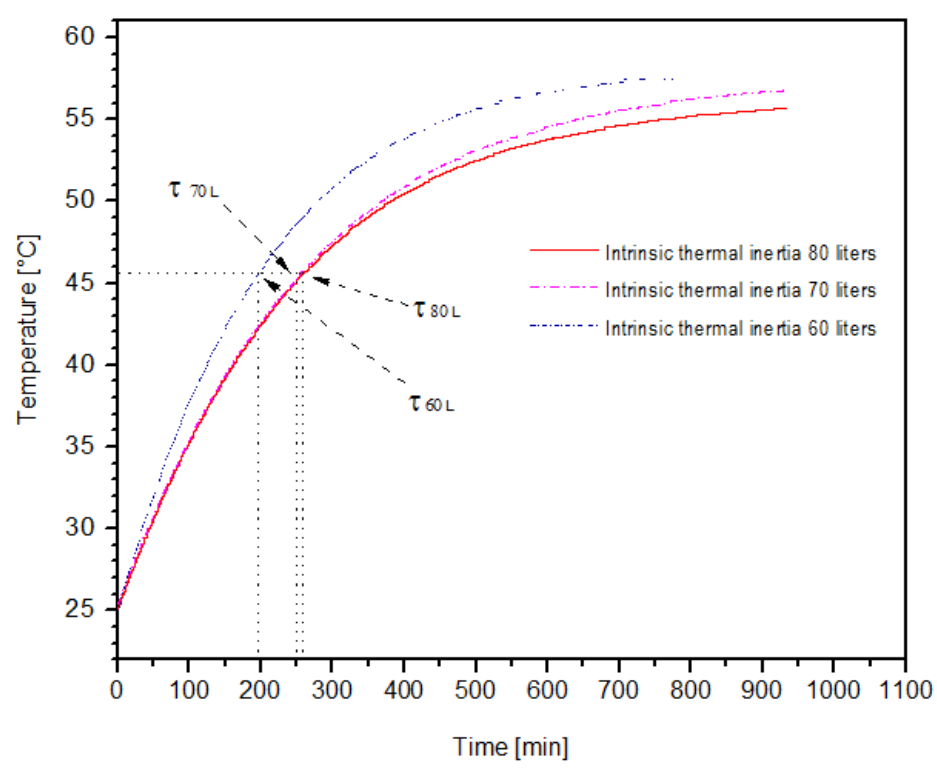

Fig. 6: The Intrinsic Thermal Inertia of the Loop Reactor for Several Water Volumes.

Where: 


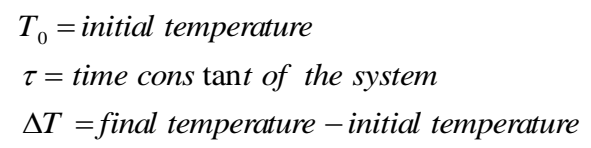

Fig. 7 shows the most optimized typical response in steady state at $60 \pm 0.2^{\circ} \mathrm{C}$ and 60 liters, applying hybrid fuzzy PID control algorithm.

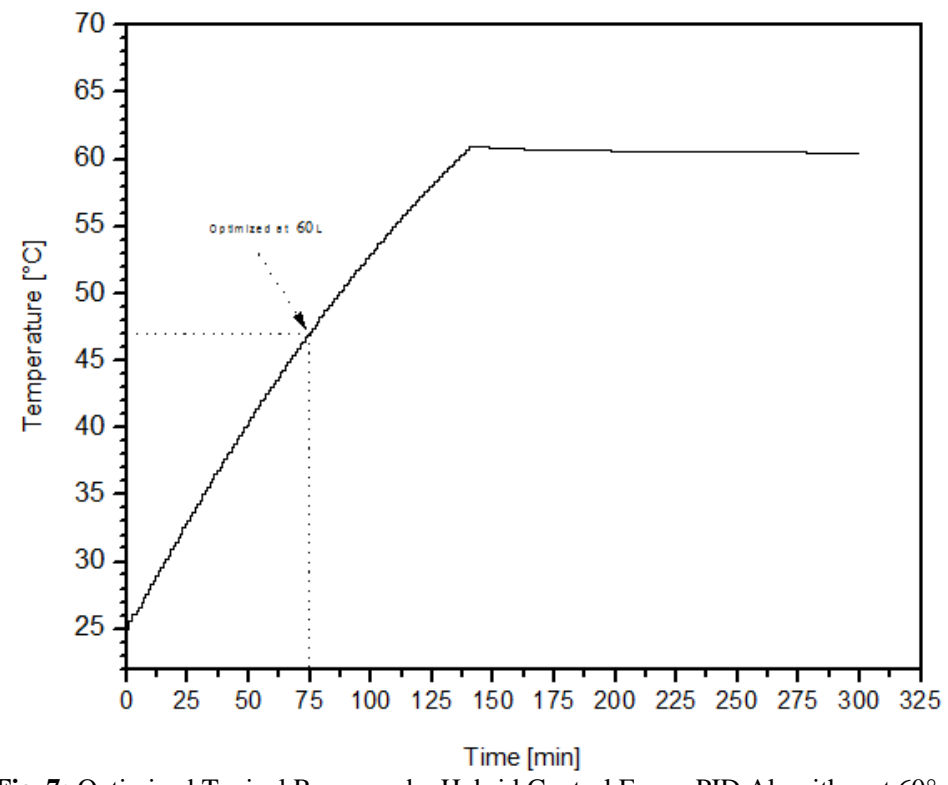

Fig. 7: Optimized Typical Response by Hybrid Control Fuzzy-PID Algorithm at $60^{\circ}$.

The time response and the overshoot were reduced $4 \%$ and $8 \%$ respectively. It is important to indicate that the specific rules, in this case, were:

Fast=much much more, Overshoot=some and Low=nothing. The PID parameters were modified, resulting $b P=0001 \%$, $t i=000.2$ minutes and $t d=0002$ seconds.

\section{Conclusions}

The temperature simplified control of a circulated flow loop reactor for microbial corrosion testing by a fuzzy-PID control algorithm was implemented. A fuzzy rule-based control was used in order to optimize the step response by using the technique called "manual tune by approximation" with a classical PID controller. Experimental tests were carried out in the loop reactor at several water volumes and several set-points of temperature, and their thermal behavior were analyzed. Accuracy in the water temperature control was crucial since the microbial culture was very susceptible to small changes in their habitat temperature. The implementation of an intelligent control allowed to reach the set-point without overshoot, then decrease the rising time by using fuzzy rules. We were able to improve the performance of the loop reactor by using this novel soft computing control technique. The results show that the optimal setting of the fuzzy-PID control algorithm applied in this paper can reduce the energy consumption and increase the performance of the thermal behavior of a loop reactor for microbial corrosion testing which can generate a good economic benefit to the industrial applications.

\section{Acknowledgements}

The authors are indebted to Mr. Fernando García for his design of the loop reactor and to M.C. Guillermo Vázquez for computational assistance.

\section{References}

[1] C. G. Hill, Chemical Engineering Kinetics and Reactor Design, John Wiley and Sons, New York, 1977.

[2] D. Zheng and K.A. Hoo, System identification and model-based control for distributed parameters systems, Computers and Chemical Engineering 28(8) (2004) 1361-1375. http://dx.doi.org/10.1016/j.compchemeng.2003.09.035.

[3] W. Zhnag and R. Zhang, Cascade fuzzy control for gas engine driven heat pump, Energy Conversion and Management 46 (2005) $1757-1766$. http://dx.doi.org/10.1016/j.enconman.2004.09.003. 
[4] H. Nahel and D. Panagiotis, Coordinating feedback and switching for control of spatially distributed process, Computers and Chemical Engineering 28 (2004) 111-128. http://dx.doi.org/10.1016/S0098-1354(03)00174-1.

[5] A.P. Melo, J.C. Pinto and E.C. Biscaia, Characterization of the residence time distribution in loop reactors, Chemical Engineering Science 56 (2001) 2703-2713. http://dx.doi.org/10.1016/S0009-2509(00)00517-0.

[6] O. Castillo, A. Alanis, M. García and H. Arias, An intuitionistic fuzzy system for time series analysis in plant monitoring and diagnosis, Applied Soft Computing 7 (2007) 1227-1233. http://dx.doi.org/10.1016/j.asoc.2006.01.010.

[7] L. Sheremetov, I. Batyrshin, D. Filatov, J. Martínez and H. Rodríguez, Fuzzy expert system for solving lost circulation problem, Applied Soft Computing, 8 (2008) 14-29. http://dx.doi.org/10.1016/j.asoc.2006.11.003.

[8] W. Wang, L. Han-Xiong and Z. Jingtao, A hybrid approach for supervisory control of furnace temperature, Control Engineering Practice 11 (2003) 1325-1334. http://dx.doi.org/10.1016/S0967-0661(02)00257-5.

[9] M. Santos and E.D Dexter, Control of a cryogenic process using a fuzzy PID scheduler, Control Engineering Practice 10 (2002) $1147-1152$. http://dx.doi.org/10.1016/S0967-0661(02)00062-X.

[10] B.M. Mohan and A. Shina, Analytical structure and stability analysis of a PID controller, Applied Soft Computing, 8 (2008) $749-758$. http://dx.doi.org/10.1016/j.asoc.2007.06.003.

[11] T. Hong and W.P Jepson, Corrosion inhibitor studies in large flow loop at high temperature and high pressure, Corrosion Science 43 (2001) 1839-1849. http://dx.doi.org/10.1016/S0010-938X(01)00002-6.

[12] A. K. Lee, M. G. Buehler and D. K. Newman, Influence of a dual-species biofilm on the corrosion of mild steel, Corrosion Science 48 (2006) 165-178. http://dx.doi.org/10.1016/j.corsci.2004.11.013.

[13] I. Perissi, U. Bardi, S. Caporali and A. Lavacchi, High temperature corrosion properties of ionic liquids, Corrosion Science 48 (2006) 23492362. http://dx.doi.org/10.1016/j.corsci.2006.06.010.

[14] M. Papagiannia and M. Matev, Desing of a tubular loop bioreactor for scale-down of fermentation processes, Biotechnology Progress 19(5) (2003) 1498-1504. http://dx.doi.org/10.1021/bp030002y.

[15] J.A. Trilleros and P. Redondo, Three-phase airlift internal loop reactor: correlations for predicting the main fluid dynamic parameters, Journal of Chemical Technology and Biotechnology 80(5) (2005) 515-522. http://dx.doi.org/10.1002/jctb.1209.

[16] R. Cheesewright, C. Clark and D.Bisset, Understanding the experimental response of Coriolis mass flow meters to flow pulsations. Flow Measurement and Instrumentation 10 (1999) 207-215. http://dx.doi.org/10.1016/S0955-5986(99)00014-X.

[17] Y. Ji and K.O. Homan, Transition from gravity-to inertia-dominated behavior computed for the turbulent stably-stratified filling of an open enclosure, International Journal of Heat and Fluid Flow 27 (3) (2006) 490-501. http://dx.doi.org/10.1016/j.ijheatfluidflow.2005.11.006.

[18] F.S. Estrada, A.C. Cleland and D.J. Cleland, Prediction of the dynamical thermal behavior of walls for refrigeration rooms using lumped and distributed parameter model, International Journal of Refrigeration 24 (2001) 272-284. http://dx.doi.org/10.1016/S0140-7007(00)00018-9.

[19] M. Galluzo, R. Ducato, V. Bartolozzi and A. Pocciotto, Expert control of DO in the aerobic reactor of an activated sludge process, Computers and Chemical Engineering 25 (2001) 619-625. http://dx.doi.org/10.1016/S0098-1354(01)00642-1.

[20] Y.T. Juang, Y.T. Chen and C.P. Huang, Design of fuzzy PID controllers using modified triangular membership functions, Information Sciences, 178 (2008) 1325-1333. http://dx.doi.org/10.1016/j.ins.2007.10.020.

[21] P. Melin and O. Castillo, Automated mathematical modeling and simulation for bacteria growth control in the food industry using artificial intelligence and fractal theory, Journal of. Systems, Analysis, Modeling and Simulations 29 (1997) 189-206.

[22] P. Melin, O. Castillo, Modeling, simulation and control of non-linear dynamical systems, Taylor and Francis Inc., New York, 2002.

[23] T. Takagi and M. Sugeno, Fuzzy identification systems and its applications to modeling and control, in: IEEE Transactions on Systems Man and Cybernetics Part A, 15 (1985) 116-132. http://dx.doi.org/10.1109/TSMC.1985.6313399.

[24] A. Hua, M. Mangold, A. Kienie and D. Gilles. Nonlinear inferential control of an autonomous periodic fixed-bed reactor, Journal of Process Control 4 (1998) 239-250. http://dx.doi.org/10.1016/S0959-1524(97)00048-6.

[25] P. Sarma, Multivariable gain-schedule fuzzy logic control of an exothermic reactor, Engineering Applications of Artificial Intelligence 14 (2001) 457-471. http://dx.doi.org/10.1016/S0952-1976(01)00003-3.

[26] H. Nahel and D. Panagiotis, Integrating robustness optimally and constraints in control of nonlinear processes. Chemical Engineering Science 56 (2001) 1841-1868. http://dx.doi.org/10.1016/S0009-2509(00)00530-3.

[27] H. Chin-an and C. Rongshun, Intelligent control of exit temperature in a gas-fuel can-type combustor, Engineering Applications of Artificial Intelligence 15 (2002) 391-400. http://dx.doi.org/10.1016/S0952-1976(02)00092-1. 